Review

\title{
Toxicology of Nanomaterials on Zebrafish
}

\author{
Jimmy De León, María del C. Cotto and Francisco Márquez \\ Nanomaterials Research Group-NRG, \\ School of Natural Sciences and Technology, Universidad Ana G. Méndez-Gurabo Campus, 00778PR, USA
}

\section{Article history}

Received: 23-02-2019

Revised: 21-03-2019

Accepted: 11-04-2019

Corresponding Author:

Jimmy De León

Nanomaterials Research

Group-NRG, School of Natural

Sciences and Technology,

Universidad Ana G. Méndez-

Gurabo Campus, 00778PR,

USA

Email: jde350@suagm.edu

\begin{abstract}
Increases in the use and the progressive manufacture of new Engineered Nanomaterials (ENMs) lead to inquire about their impact on the environment. Due to the small size, high reactivity inside organisms and the unusual physicochemical properties of the ENMs, the predictions of toxicity are very complex. The zebrafish (Danio rerio) has been granted as a practical alternative to study the toxicity of ENMs. In this article the toxic effects of silver nanoparticles, titanium oxide nanoparticles, zinc oxide nanoparticles, carbon nanotubes, copper nanoparticles, gold nanoparticles, cadmium nanoparticles and nano plastics were reviewed trough the most recent literature available. Every ENMs should be studied in depth independently, considering co-exposures, environmental matrices, the effects of variations in size and concentrations, the potential effects of prolonged exposure, the coverage of ENMs, specific organism and targeted organs. This information will help to identify deficiencies in research trends and reinforce the safest ways to use ENMs.
\end{abstract}

Keywords: Zebrafish, Toxicity, Nanomaterial, Copper, Gold, Silver, Titanium Oxide, Zinc Oxide, Carbon Nanotubes, Cadmium, Nanoplastics, Environmental Impact

\section{Introduction}

The Engineered Nanomaterials (ENMs) are very innovative products that have a wide field of utilities, so they can easily access the environment. The properties of ENMs are as different as their composition, doing predictions of destination and risk assessment very complex. The physicochemical properties of nanomaterials are not enough to estimate organisms risks; it is necessary to consider other aspects including matrix, ions, impurities, physical and chemical parameters and the entire ecosystem as a whole. The aquatic environment is one of the matrices with more probability to be affected by ENMS and it is also very difficult challenge to predict. Aquatic systems can interact with nanomaterials and transform them by dissolution, aggregation, sedimentation and photoreactions, among others (Vale et al., 2016). The reactions between ENMs and environmental pollutants are also a cause for concern. In cell-free systems the most usual interaction between ENMs and pollutants is the adsorption, based on hydrophobic effects, hydrogen bonds, ion exchange and pi-pi bonding and covalent and electrical interaction (Liu et al., 2018). Under cellular and molecular approach, ENMs within the organism can modify bioaccumulation and affect the biodistribution and metabolism of pollutants (Liu et al., 2018). Some interactions may produce mitigations of toxic mechanisms in specifics organisms. Dissolved natural organic matter, humic substance, carbohydrates and proteins can play an important role in reducing the adverse effects of ENMs released in aquatic environments (Kteeba et al., 2017).

The ENMs are increasing their environmental applications in different processes, such as water and waste water treatment, due to their properties of high surface area, energy cost and energy efficiency, ease of regeneration and chemical activity, among others (Gautam et al., 2019). The efficiency of ENMs in certain processes, for example remediation of contaminated water, is clearly established, although the uses in the free environment are subjects of great concern. Fate predictions of ENMs, especially on aquatic systems, are quite difficult and, until now, modeling is restricted to the processes of aggregation and dissolution (Williams et al., 2019). Each nanomaterial has specifics properties that can change according to the conditions and, even more, the same ENM can vary its effects by size and concentrations.

\section{Biological Models and "Nanotoxicology"}

Studies have been underway for a number of years to determine the potential toxicity of specific nanomaterials and, until now, the researchers conclude with the need to generate new assessment and strategies of evaluation. Approaches as in silico technics emerge as essentials 
methods for risk assessments that undoubtedly have their advantages. Nanotoxicity studies, developed using computational methods, have the potential to be rapid and inexpensive screening tests, allowing the categorization of materials by toxic levels (Oksel et al., 2015). ENMs toxicological modeling faces some crucial issues, i.e., experimental data deficiency, lacks of common metrics, the need for standardized toxicological protocols and validated methods, instability of small particles that produce bulk materials by agglomeration and the complexity of creating a complete database of attributes to predict toxicity across the great diversity of ENMs (Oksel et al., 2015). Most of the research concludes on the need to develop research that contributes with quality data to the characterization of the toxic effect of ENMs. The use of biological models is presented as a useful method to investigate the behavior of ENMs, providing empirical information to improve modeling, methodologies and materials manufacturing. The in vivo toxicity models commonly applied to evaluate nanomaterials include Daphnia magna, zebrafish, rats, $\mathrm{C}$. elegans, Drosophila and artemia, among others.

\section{Zebrafish as Biological Model}

Danio rerio, or zebrafish, is a well-known model organism studied since 1960's (Kalueff et al., 2014). This organism has several characteristics that make it suitable for environmental toxicology research, starting with the economic feasibility, in comparison with others biologicals models of vertebrates. As shown in Fig. 1, due to the relatively small size of the adult fish, studies with a large number of samples are feasible. The external fertilization and transparency during development are useful characteristics to toxicological studies. More important is that $\mathrm{D}$. rerio is very homologue to humans; they have chambers and rhythmically pump oxygen-carrying blood through the body; they have eyes with retinal structure, liver, pancreas, kidneys and intestines, backbone and more than $80 \%$ of their genes have human counterpart (FDA, 2013). A large part of the studies with $D$. rerio focus on teratogenic and development effects of substances on the larvae and on the fry. Common use to assess the toxic effect on development is due to rapid reproduction, embryos are available throughout the year, transparency of the larvae body, or easy breed, among others. D. rerio is now used to evaluate the potential toxic effects of ENMs.

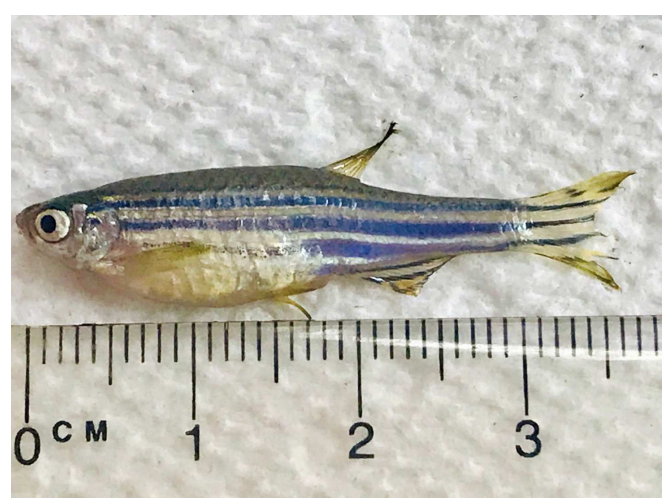

Fig. 1: Medium size adult zebrafish (D. rerio.)

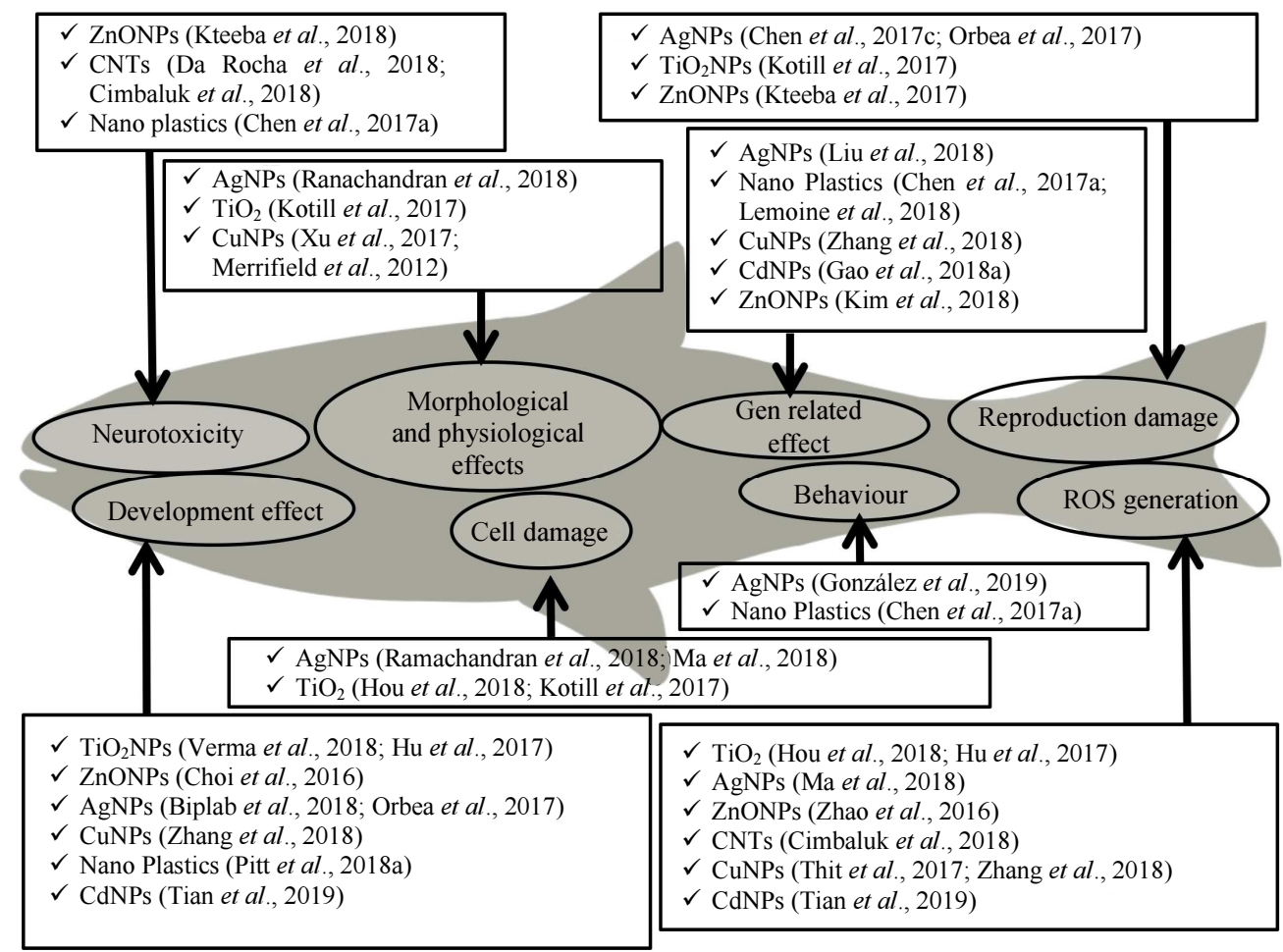

Fig. 2: Toxic effects of ENMs on D. rerio according to references cited in this article 
Studies confirm that some ENMs share the same adverse effects on the zebrafish as show in Fig. 2. Mixtures and variations in ENMs properties could have the worst effects.

\section{Silver Nanoparticles}

Silver nanoparticles (AgNPs) are materials widely used in the development of antibacterial, paints, textiles, cosmetics, personal care products and supplements, among others. It is perhaps the most ENM used in commercials products, so it is more likely to be found in the environment. The inconsistency in the research results leads the scientific community to debate between the potential danger and the empiric evidence of the AgNPs effects. The effects depend on particle sizes, aggregation, concentrations, stages of growth of the organism, accumulation organs or tissues and the matrix where the AgNPs can interact. A bioaccumulation study by Sung et al. (2018) used zebrafish of ca. $2 \mathrm{~cm}$ long, to shortly exposed them to Au and AgNPs of $30 \mathrm{~nm}$ and $80 \mathrm{~nm}$. This research found that larger AgNPs accumulated in the liver and intestine tissue, while smaller particles were found in the gills. However, since the intestine has the largest surface area, the absorption is greater and the effect is more toxic compared to the gills and muscles (Liu et al., 2019). It has been proposed that the main toxicity mechanism of AgNPs in gills and intestine is due to a malfunction of related genes encoding enzymes and proteins, leading to irregularities in DNA replication, protein removal and metal ion binding (Liu et al., 2009). Bioaccumulation of AgNPs in zebrafish through the food chain was also evaluated by transferring brine shrimps exposed, producing as a result the metal accumulation in the liver and intestine, causing deterioration in the health of the fish (Lacave et al., 2017). AgNPs bioaccumulate in gonads tissues, causing an increment of Reactive Oxygen Species (ROS), inducing germ cell apoptosis (Ma et al., 2018). Cambier et al. (2018) could not find survival neither survival adverse effects in 6 days post fertilization larvae exposed to AgNPs through 15 days. The same study observed accumulation mainly on the liver blood vessels and in the interstitial tissue between the intestine and liver (Cambier et al., 2018). Bioaccumulation can be altered by the interaction of AgNPs with environmental matrices and change the risk potential. Gupta et al. (2016) showed that AgNPs can be adsorbed on clays and this complex presents higher mortality rates for zebrafish embryos compared to bare AgNPs.

On the effects of reproduction and development, AgNPs has promoted different opinions of the counterparts. At reproduction levels, Chen et al. (2017c) compared the effects of $\mathrm{AgNPs}$ and $\mathrm{AgNO}_{3}$ as control and found apoptosis in ovarian follicle cells surrounding the oocyte in both cases, but the genes related to oxidative stress were more sensitive to the particles. Zebrafish from 24 to $120 \mathrm{~h}$ after fertilization (hpf) was approximately 200 times less sensitive to AgNPs, as compared to Ag ions and the main toxic mechanism of this acute exposure was the inhibition of $\mathrm{Na}^{+}$uptake (Boyle and Goss, 2018). Sarkar et al. (2018) propose AgNPs as a growth-stimulating agent in low doses because they found highest hatching success in low doses of nanoparticles compared to control. At higher doses, the same research reports toxic effects in embryos and larvae, including bending in myotome, deformity in tail region, somites, notochord and swelling in anterior and posterior region of embryos and larvae (Sarkar et al., 2018). Embryos exposed after fertilization (4-120 hpf) at concentrations between 0.03 to $3 \mathrm{mg} / \mathrm{L}$ of AgNPs, showed hyperactivity but did not present dangers for hatching, morphology, or mortality (González et al., 2018).

Adult zebrafish exposed to $10 \mu \mathrm{g} / \mathrm{L}$ of AgNPs reduced fertility and increased malformation prevalence in the embryos only after two weeks of exposure (Orbea et al., 2017). At the half of the $\mathrm{LC}_{50}$ of AgNPs during 14 days of exposure, zebrafish exhibit many negative effects, such as higher levels of ROS generations and morphological repercussions in liver and gills as cell membrane damage, irregular cell outlines, pyknotic nuclei and complete disruption of cells (Ramachandran et al., 2018).

As shown, the AgNPs have a huge potential risk to the environment and aquatic organisms. All applications and advantages presented by this material will continue to increase, so special attention must be paid through additional studies.

\section{Titanium Oxide Nanoparticles}

Titanium Oxide Nanoparticles $\left(\mathrm{TiO}_{2} \mathrm{NPs}\right)$ are considered as one of the most industrially used ENMs due to its wide variety of applications. The uses of $\mathrm{TiO}_{2} \mathrm{NPs}$ include among others: Sunscreens, paints and coatings, inks, plastics, papers, or catalysts, which results in an annual production of more than 10,000 Tm worldwide, with huge growth projections (Drobne, 2018). Due to this massive use, it is very likely that this ENM ends up being released into the environment and can lead to significant accumulations in soil or water. Some studies found potentially adverse effects and defined three main mechanisms of action in organisms: The production of ROS, the peroxidation of cell membrane lipids and cell wall damage and the incorporation of $\mathrm{TiO}_{2} \mathrm{NPs}$ into cellular organelles and macromolecules (Hou et al., 2018). In zebrafish, Kotil et al. (2017) observed that doses of 4 $\mathrm{mg} / \mathrm{L}$ of $\mathrm{TiO}_{2} \mathrm{NPs}$ induced autophagy and necrosis in Sterli cells, therefore spermatogenic cells and testicular morphology were affected. Chen et al. (2018b) exposed zebrafish parents to $\mathrm{TiO}_{2} \mathrm{NPs}$ and Bisphenol A (BPA) in mixtures and independently and found that the larvae 
from fish exposed to $\mathrm{TiO}_{2} \mathrm{NPs}$ showed alterations of the metabolic pathway of glucose. This study is one of the few available on effects with adult fish. The mixture caused severe inhibitions in growth and survival of the first-generation larvae, altering activity of phagosomes and lysosomes (Chen et al., 2018b). The simultaneal exposure of $\mathrm{TiO}_{2} \mathrm{NP}$ and BPA leads to a change in the intestinal microbial community of the adult zebrafish and to the generation of oxidative stress (Chen et al., 2018a). As others ENMs there is a concern about the easy mobility of these small materials through the body of an organism and through their cells, but more important is the ability to transport others substances to the cells or internal organs. Zebrafish exposed to a combination of $\mathrm{TiO}_{2} \mathrm{NP}$ and tris(1,3-dichloro-2-propyl) phosphate (TDCIPP) resulted in an increase in the bioaccumulation of TDCIPP, affecting females, reducing certain hormones, inhibiting eggs productions and promoting the developmental toxicity in first-generation larvae (Ren et al., 2018). Molecules or ions in presence of ENMs may affect organisms, although under normal conditions there would be no danger.

Additionally, different studies focus on the effects of $\mathrm{TiO}_{2} \mathrm{NPs}$ on the development of $\mathrm{D}$ rerio embryos due to the sensitivity of the organism and the divergent results. A different conclusion about ROS has been developed by Verma et al. (2018), since they claim that $\mathrm{TiO}_{2} \mathrm{NPs}$ enhance the scavenging of ROS, although it promotes deformities on embryos head trunks and tail. One-hour embryos co-exposed for 96 hours to $\mathrm{TiO}_{2} \mathrm{NPs}$ and multiwalled carbon nanotubes show no acute toxicity, although single exposure to $100 \mathrm{mg} / \mathrm{L}$ of $\mathrm{TiO}_{2} \mathrm{NPs}$ affected the total length of the embryo (Da Silva et al., 2018). Similar results were found by $\mathrm{Hu}$ et al. (2017), that after exposing the embryos, they observed an increase in the rate of malformations and the decrease in the incubation times, while the mortality remained a control group. This study also confirms the bioaccumulation in the brain, the cell death of the hypothalamus and the generation of ROS contrary to the findings of Verma et al. (2018).

\section{Zinc Oxide Nanoparticles}

Zinc Oxide nanoparticles (ZnNPs) play an important role in environmental, energy and medical applications. ZnONPs are also used together with polymer nanocomposites to manufacture fuel cells, supercapacitors, energy harvesting, biomedical applications, antibacterial activities, sensors and optical, magnetic and electromagnetic protection (Ponnamma et al., 2019). With such broad functions and uses, the likelihood of environmental contamination is significant and the effects on organisms as zebrafish should be thoroughly investigated. ZnONPs have been proposed as a remediation substance for aquatic systems and it is important to fully understand the potential worst toxic effects due to the nanoparticles, avoiding aggravating pollution with even more dangerous substances. Electrospun polyacrylonitrile sheets as support of $\mathrm{ZnO}$ nanocrystals have been used to degrade diethyl (4nitrophenyl) phosphate, resulting in a product with less toxicity impact on zebrafish (Lakshmi et al., 2019). On other hand, Misra et al. (2018), developed ZnONPs materials doped with $\mathrm{Fe}$, impregnated on Kaolinite $(\mathrm{ZnO} / \mathrm{K})$, with the purpose of disinfecting the water in presence of zebrafish. Adult zebrafish were exposed to $\mathrm{FeZnO} / \mathrm{K}$ and a photocatalytic disinfection of an enteric multidrug resistant bacteria was performed successfully without causing any intestinal damage to the fish. As expected, environmental matrices can interfere in the behavior and effects of ENMs on the organisms. The dissolved organic material can mitigate some effects of ZnONPs, even reverse abnormalities in phenotypes (Kteeba et al., 2018). However, ZnONPs can cause abnormalities in secondary motoneuron axon phenotypes until the second generation (Kteeba et al., 2018). Research on co-exposure of ENMs and other pollutants is also necessary and has been object of study because the mixtures outcomes can be magnified. First, the toxicity to aquatic organisms may be more significant from $\mathrm{ZnONPs}$ than the effects from dissolved $\mathrm{Zn}$ ions by themselves (Ye et al., 2018). Co-exposure of ZnONPs with perfluorooctane sulfate increases the effects on oxidative stress and apoptosis that independent exposures (Du et al., 2017). Zebrafish chronically exposed during development to ZnONPs and perfluorooctane sulfonate shows limited growth in body length and weight, less spawning and higher mortality. In addition, these effects are transferred to the first generation as less fertilization, less hatching, increase in mortality and malformations (Du et al., 2018).

The toxicity of ZnONPs has been studied and, as analyzed in other ENMs discussed in this article, the generation of ROS is one of the most well-known mechanisms of action, but it is not the only effect. Toxic effects on zebrafish were determined by exposure to sublethal concentrations of ZnONPs, among them several key genes related to cancer were activated, in addition to the induction of cell differentiation and pathways associated with immune system (Kim et al., 2016).

As any potential toxic substance, the main concern of the ZnONPs is the potentially adverse effect on development. Embryo hatching was reduced to $0 \%$ by the exposure to $50 \mathrm{mg} / \mathrm{L}$ of $\mathrm{ZnONP}$ at $72 \mathrm{hpf}$, while the same concentration at $96 \mathrm{hpf}$ reduced hatching to $20 \%$ (Kteeba et al., 2017). Embryos showed pericardial edema, tail edema and yolk-sac edema when exposed to concentrations as low as $0.01 \mathrm{mg} / \mathrm{L}$ of ZnONPs (Choi et al., 2016). If the embryos (96 hpf) are exposed to ZnONPs, oxidative stress is induced, increasing the percentage of apoptotic cells (Zhao et al., 2016). 


\section{Carbon Nanotubes}

Carbon nanotubes (CNTs) are a novel and popular material widely studied and applied. The physical and mechanical properties of the CNTs allow applications in the development of sensors, filtration processes, new fabrics, nanorobots, drugs, batteries and energy, among others (Kumar et al., 2019). The ecotoxicological effect of the CNTs is still under study, as occurs with the others ENMs. Zebrafish, as a model organism with human homology, attends both the ecotoxicological concern and the starting point of the risk assessments for human health. As at the moment there is not an excessive amount of CNTs in the environment, science must look for chronic and neurological effects and not just focus on high concentration expositions. Zebrafish was exposed to $10 \mu \mathrm{L}$ dose of $30 \mathrm{mg} / \mathrm{kg}$ suspension of single walled CNTs (SWCNTs), which resulted in a sixfold increase in brain levels of dopamine and serotonin, while reducing the activity of acetylcholinesterase (AchE) activity (Da Rocha et al., 2018). Cimbaluk et al. (2018), found subchronic neurotoxicity in zebrafish by decreasing the dose-response in AchE activity and the induction of oxidative stress confirmed by the increased activity of superoxide dismutase and catalase (Cimbaluk et al., 2018).

The main objectives of the current ENMs toxicological research are to find effects when the substances are in the same matrix. Zebrafish has been exposed to different substances in presence of CNTs to assess changes in the effects of organisms due to the mixtures. Cadmium in the presence of oxidized carbon nanotubes (oxMWCNT) was more toxic at low concentrations and induced apoptosis and necrosis in in vitro tests of zebrafish liver cells (Morozesk et al., 2018). The co-exposure of MWCNTs with fluoxetine leads to an increase in bioavailable accumulation of 46$99 \%$, leading to an increase in metabolite formation (Yan et al., 2018a). The findings suggest that, once again, adverse effects with more abundant substances and ENMs can be exacerbated or mitigated. Another co-exposure experiment determined that SWCNTs decrease the accumulation of Perfluorooctane Sulfonate (PFOS) in liver, intestines, gills and brain of zebrafish but increased it in the skin, suggesting changes in bioavailability of PFOS (Li et al., 2017). Simultaneous exposure increased ROS generation and enhanced the AchE activity, catalase and superoxide dismutase (Li et al., 2017). Wang et al. (2017) found that MWCNTs protect early stages of the zebrafish against PFOS, which affects the hatching rate, heart rate and body length. Contrary to other studies, they observed decreases in ROS levels, the activity of superoxide dismutase and catalase. Moreover, since zebrafish has useful characteristics during development, research on co-exposures focuses on the early stages of the fish.
SWCNTs functionalized with polyethylene glycol are toxic without being absorbed by the organism and the effects are shown in the mortality, the delay of the hatching, malformations, reductions in body length, the increase in ROS generation and DNA damage (Cordeiro et al., 2018). Otherwise 3-4 hpf zebrafish embryos show protective effects of MWCNTs by reducing the expected estrogenic response of $17 \beta$ estradiol exposures (Yan et al., 2018b).

\section{Copper Nanoparticles}

Copper nanoparticles (CuNPs) are used in multiple applications, including antibacterial/antimicrobial, catalysis, medical purposes, electrochemical processes, among others. Is highly probable that CuNPs impact the environment through all its applications wastes. CuNPs in environment can be easily transformed into $\mathrm{CuS}$ and other insoluble forms of $\mathrm{Cu}$ (Keller et al., 2017). It is suggested that the order of ecological toxicity of the $\mathrm{Cu}$ compounds is as follows: Copper II $>$ nano $\mathrm{Cu}^{0}>$ nano $\mathrm{Cu}(\mathrm{OH})_{2}>$ nano $\mathrm{CuO}>$ micro $\mathrm{Cu}$ compounds and the effects at lower concentration are found in aquatic organisms (Keller et al., 2017). Once the CuNPs enter the free environment, they will interact with other substances based on $\mathrm{Cu}$ ions or $\mathrm{Cu}$ compounds. The independent exposure of $0.25 \mathrm{mg} / \mathrm{L}$ of CuNPs and $0.25 \mathrm{mg} / \mathrm{L} \mathrm{Cu}^{+2}$ ions obstructs the inflammation in swimming bladders of zebrafish embryos (Xu et al., 2017). Both copper ions and CuNPs affect ROS production, but in general $\mathrm{Cu}$ ions have toxic effects at lower concentrations than CuNPs in zebrafish and fry (Thit et al., 2017). The median lethal concentration of CuNPs at $96 \mathrm{~h}$ of exposure in zebrafish larvae are $242.4 \mu \mathrm{g} / \mathrm{L}$ and $85.73 \mu \mathrm{g} / \mathrm{L}$ of $\mathrm{CuCl}_{2}$, moreover the bioaccumulation of $\mathrm{Cu}$ is higher when co-expose to CuNPs (Chen et al., 2011). A comparison in the effects on embryos from CuNPs and $\mathrm{Cu}$ exposures, enhance hemoglobin and increase ROS generation, although CuNPs show more increases in vascular endothelial growth factor signaling and expression of vessel endothelial marker flil (Zhang et al., 2018).

CuNPs interfere in the intestinal and digestive gut of zebrafish. Zebrafish that were exposed through food to CuNPs eradicate the intestinal microbes to undetectable levels (Merrifield et al., 2013). Moreover effects of CuNPs on embryos were also found, as eye hypoplasia and relatively absence of digestive gut (Zhang et al., 2018).

\section{Gold Nanoparticles}

Gold Nanoparticles (AuNPs) are widely used in a long list of different applications, as for example photoelectrochemistry, catalysis, sensor and medical applications, including imaging, therapy, or biosensors, among others (Caballero-Díaz and Valcárcel, 2014). Considering all the ENMs, AuNPs have the least empiric 
evidence of adverse effects in organisms until now, although it is also true that there are very few studies on in vivo toxicity (Caballero-Díaz and Valcárcel, 2014). In vitro, some of the mechanisms reported are: Genotoxicity, generation of ROS, mitochondrial damage, apoptosis, leakage of toxic materials, endocrine disruption, interactions with lipids and proteins, altered gene expression and cellular morphology changes (Caballero-Díaz and Valcárcel, 2014). The greatest number of experiments establishes the low or no toxicity for zebrafish. Embryos exposed to $100 \mu \mathrm{L} / \mathrm{mL}$ of gold nanoclusters show no toxic effects on survival, hatching rate, heart rate, malformations and gene expression (Chandirasekar et al., 2016). No hazard was found for zebrafish, but the soluble $\mathrm{Au}$ may be toxic to other organisms (García-Cambero et al., 2013). In comparison with AgNPs, the AuNPs have less bioaccumulation in liver tissue and intestines, but the particle size is decisive for accumulation in target organs (Sung et al., 2018). On the other hand, a study found that the AuNPs were not toxic to zebrafish embryos and that bioaccumulation is similar to AgNPs (Asharani et al., 2010).

Toxic effects are found at very high concentration, which may be far from environmental relevance. AuNPs used as anticancer agents show 100\% embryo mortality at $300 \mathrm{mg} / \mathrm{mL}$ (Ramachandran et al., 2017). Embryos exposed to concentrations ranging from 0.08 to $50 \mathrm{mg} / \mathrm{L}$ of AuNPs, functionalized with $\mathrm{N}, \mathrm{N}, \mathrm{N}-$ trimethylammoniumeethanethiol end in cell death of the eye, smaller and malpigmented eyes, hypoactive swimming behavior and axonal growth inhibition (Kim et al., 2013). It is very likely that the effects of AuNPs are more related to the Au effects than the particle itself. Acute exposure to dissolved $\mathrm{Au}$ generates temporary changes in the behavior and swim performance (Strungaru et al., 2018). The progress of these investigations must necessarily be carried out through co-exposures with $\mathrm{Au}$ and other compounds in order to assign the real risk of AuNPs.

\section{Nanoplastics}

In recent years, questions about the impact on the environment and ecosystems of micro and nanoplastics have been rising among scientists. Materials at the nanoscale can be introduced into the environment as larger particle degradation products or by ENMs of polymers. The microscale plastics are more studied, although at the nanoscale they continue to emerge continuously. At the environment, contaminants could be adsorbed to the microplastics, however experimentally Sleight et al. (2017) could not found increases in bioavailability of phenanthrene and $17 \alpha$-ethinylestradiol due to microplastics actions, although a reduction in zebrafish larvae was observed. Micro plastics accumulate in the gastrointestinal tract and induce changes in gene expression of zebrafish lavae (LeMoine et al., 2018). Effects on zebrafish larvae were greater by nanoplastic than microplastics (Chen et al., 2017a). When nanoplastics reach environment can be introduced in aquatics organisms through ingestion or oral exposure, dermal exposure, transference from previous generation, or biomagnification, among others. The uptake of Polystyrene Nanoparticles (PSNPs) by zebrafish is primary, through oral exposures, in comparison to dermal; however, exposures through both routes show higher abortions (Van Pomeren et al., 2017). Bioacumulation of PSNPs in zebrafish embryos are observed in yolk sac, gastrointestinal tract, gall bladder, liver, pancreas, heart and brain (Pitt et al., 2018a). Firstgeneration embryos, that have not been directly exposed, accumulate PSNPs in the yolk sac, gastrointestinal tract, liver and pancreas (Pitt et al., 2018b). The PSNPs penetrate the chorion and accumulate in all body but more in lipid regions (Lee et al., 2019).

Nanoplasctics show toxicity mainly in the early stages of zebrafish. Nanoplasctics have adverse effects on zebrafish larvae, as shown by Chen et al. (2017c), that observed a reduction in larval locomotion during darkness, a reduction in body length by $6 \%$, inhabitation of acetylcholinesterase activity and upregulated gene expressions (Chen et al., 2017a). PSNPs exposures during development reduce glutathione reductase activity in brain, muscle and testes and in the stages of larvae decrease the heart rate and generate hypoactivity when swimming (Pitt et al., 2018a). Even the first generation acquired bradycardia and reduced the level of thiols and the activity of glutathione reductase (Pitt et al., 2018b).

When nanoplastics are mixed with other substances, the adverse effects can be exacerbated in many ways. Co-exposures of nanoplastics with $2000 \mathrm{mg} / \mathrm{L}$ of $17 \alpha-$ ethynylestradiol (EE2), increases the adsorption for EE2 and enhances hypoactivity (Chen et al., 2017a). Bisphenol A in co-exposure with nanoplastics increases the accumulation by 2.2 and 2.6 times in the head of zebrafish and viscera, which also accentuates neurotoxicity in the central nervous system and the dopaminergic system (Chen et al., 2017b). Embryos exposed to PSNP with Au compared to PSNPs alone, generate the intensification of adverse effects for survival, hatching, developmental anomalies and cell death (Lee et al., 2019).

\section{Cadmium Nanoparticles}

Cadmium Nanoparticles (CdNPs) have a very complex behavior in the organisms, so the mechanisms of toxicity are not fully established. Recently, studies of toxicity with $\mathrm{Cd}$ in zebrafish have been conducted and the results are allowing to establish the possible mechanisms. It has been observed that the effects of CdNPs can be correlated with the effects of $\mathrm{Cd}$ ions 
$\left(\mathrm{Cd}^{+2}\right)$. Nanomaterials based on $\mathrm{Cd}$ and $\mathrm{Cd}^{+2}$ have been found to be responsible for the overexpression of six genes, in addition to the reduced expression of another 27 in the zebrafish (Gao et al., 2018a). $\mathrm{Cd}^{+2}$ ions have more potential of bioaccumulation than cadmium selenide quantum dots although with a homogeneous distribution along the surface of zebrafish larvae (Zarco-Fernández et al., 2016). Hydroxyapatite-loaded CdNPs with $\mathrm{Cd}^{+2}$ can generate increases in the activities of superoxide dismutase, peroxidase and catalase enzymes; furthermore it can damage liver DNA (Gao et al., 2018b). By itself quantum dots (QDs) retard hatching and increase the oxidative stress of embryos, as evidenced by exposure to mercaptopropionic acid-CdTe QDs and mercaptopropionic CdS-CdTe QDs (Tian et al., 2019).

As with others ENMs, the complexity of the environment affects the actions of the materials and the possible threat to the organism. In the case of small particles, dilution expectations are not always met. Carboxilated $\mathrm{CdSe} / \mathrm{ZnS}$ QDs can agglomerate in deep well water and on the surface of fish embryos (Rotomskis et al., 2018). The damaging potential of an ENM is inherent to the particle, but modifications can be done to decrease or delay it. For example, possible carbon coatings on $\mathrm{Cd}$ nanoparticles can reduce toxicity by mitigating the release of $\mathrm{Cd}^{+2}$ (Balmuri et al., 2017).

\section{Conclusion}

ENMs are extraordinarily useful and are becoming essential for health and technology. Facing this reality, it is essential to know everything about the impact on the environment and ecosystems. Every ENM has different properties that change over time as they interact with other substances and penetrate protective barriers. Coexposure studies of ENMs with other contaminants frequently conclude in the increment of bioconcentration of the chemicals; however this is not always the case (Naasz et al., 2018). Some studies showed a lower bioavailability when co-exposures are with ENMs. Small modifications of the chemical structure of ENMs may generate changes in their reactions, increasing significantly their toxicity and turning unmanageable to reach conclusions (Kabir et al., 2018). Some materials act as Trojan horses to other dangerous species. All these challenges make evident the urgency of generate new research, focused on the in vivo potential effects by the most produced ENMs.

Concentrations of environmental relevance are not commonly used, a fact that leads to one of the main criticisms that high concentration studies have no current relevance. Additional studies should be aimed at understanding sub-lethal effects and long-term exposures, evaluating natural pathways, including plants through small organisms. Every ENM should be studied in depth to evaluate the physical and chemical interactions in different environmental matrices, the effects of variations in size and concentrations, the potential effects of prolonged exposure, the coverage of ENMs, specific organisms and targeted organs. Nanomaterials are helping and, in the near future, will help even more to solve many of society's problems. That is why we must foresee the possible effects, to establish the necessary control mechanisms.

\section{Acknowledgement}

Jimmy de León thanks the US Department of Energy and Consortium for Integrating Energy Systems in Engineering and Science Education (CIESESE) for a postgraduate scholarship.

\section{Funding Information}

Financial funding from the US Department of Energy and the Consortium for Integrating Energy System in Engineering and Science Education, CIESESE (DENA0003330), is gratefully acknowledged.

\section{Author's Contributions}

All authors contributed equally to this work.

\section{Ethics}

This article is original and contains unpublished material. The corresponding author confirms that all of the other authors have read and approved the manuscript and no ethical issues involved.

\section{References}

Asharani, P.V., Y. Lianwu, Z. Gong and S. Valiyaveettil, 2010. Comparison of the toxicity of silver, gold and platinum nanoparticles in developing zebrafish embryos. Nanotoxicology, 5: 43-54.

DOI: $10.3109 / 17435390.2010 .489207$

Balmuri, S.R., U. Selvaraj, V.V. Kumar, S.P. Anthony and A.M. Tsatsakis et al., 2017. Effect of surfactant in mitigating cadmium oxide nanoparticle toxicity: Implications for mitigating cadmium toxicity in environment. Environ. Res., 152: 141-149. DOI: 10.1016/j.envres.2016.10.005

Boyle, D. and G.G. Goss, 2018. Effects of silver nanoparticles in early life-stage zebrafish are associated with particle dissolution and the toxicity of soluble silver. NanoImpact, 12: 1-8.

DOI: $10.1016 /$ j.impact.2018.08.006

Caballero-Díaz, E. and M. Valcárcel, 2014. Chapter 5Toxicity of Gold Nanoparticles. In: Comprehensive Analytical Chemistry, Varcárcel, M. and A. LópezLorente (Eds.), Elsevier, Oxford, UK, pp: 207-254. 
Cambier, S., M. Røgeberg, A. Georgantzopoulou, T. Serchi and C. Karlsson et al., 2018. Fate and effects of silver nanoparticles on early life-stage development of zebrafish (Danio rerio) in comparison to silver nitrate. Sci. Total Environ., 610-611: 972-982.

DOI: $10.1016 /$ j.scitotenv.2017.08.115

Chandirasekar, S., C. Chandrasekaran, T. Muthukumarasamyvel, G. Sudhandiran and N. Rajendiran, 2016. Biosurfactant templated quantum sized fluorescent gold nanoclusters for in vivo bioimaging in zebrafish embryos. Colloids Surfaces B: Biointerfaces, 143: 472-480. DOI: $10.1016 /$ j.colsurfb.2016.03.067

Chen, D., D. Zhang, J.C. Yu and K.M. Chan, 2011. Effects of $\mathrm{Cu}_{2} \mathrm{O}$ nanoparticle and $\mathrm{CuCl}_{2}$ on zebrafish larvae and a liver cell-line. Aquatic Toxicol., 105: 344-354. DOI: 10.1016/j.aquatox.2011.07.005

Chen, L., Y. Guo, C. Hu, P.K.S. Lam and J.C.W. Lam et al., 2018a. Dysbiosis of gut microbiota by chronic coexposure to titanium dioxide nanoparticles and bisphenol A: Implications for host health in zebrafish. Environ. Pollut., 234: 307-317. DOI:10.1016/j.envpol.2017.11.074

Chen, L., C. Hu, Y. Guo, Q. Shi and B. Zhou, 2018b. $\mathrm{TiO}_{2}$ nanoparticles and BPA are combined to impair the development of offspring zebrafish after parental coexposure. Chemosphere, 217: 732-741.

DOI: 10.1016/j.chemosphere.2018.11.052

Chen, Q., M. Gundlach, S. Yang, J. Jiang and M. Velki et al., 2017a. Quantitative investigation of the mechanisms of microplastics and nanoplastics toward zebrafish larvae locomotor activity. Sci. Total Environ., 584-585: 1022-1031.

DOI: $10.1016 /$ j.scitotenv.2017.01.156

Chen, Q., D. Yin, Y. Jia, S. Schiwy and J. Legradi et al., $2017 \mathrm{~b}$. Enhanced uptake of BPA in the presence of nanoplastics can lead to neurotoxic effects in adult zebrafish. Sci. Total Environ.ent, 609: 1312-1321. DOI: 10.1016/j.scitotenv.2017.07.144

Chen, S.X., X.Z. Yang, Y. Deng, J. Huang and Y. Li et al., 2017c. Silver nanoparticles induce oocyte maturation in zebrafish (Danio rerio). Chemosphere, 170: 51-60. DOI: 10.1016/j.chemosphere.2016.12.016

Choi, J.S., R.O. Kim, S. Yoon and W.K. Kim, 2016. Developmental toxicity of zinc oxide nanoparticles to zebrafish (Danio rerio): A transcriptomic analysis. PLoS One, 11: e0160763-e0160763. DOI: 10.1371/journal.pone.0160763

Cimbaluk, G.V., W.A. Ramsdorf, M.C. Perussolo, H.K.F. Santos and H.C. Da Silva-De Assis et al., 2018. Evaluation of multiwalled carbon nanotubes toxicity in two fish species. Ecotoxicol. Environ. Safety, 150: 215-223.

DOI: 10.1016/j.ecoenv.2017.12.034
Cordeiro, M.F., F.A. Girardi, C.O.F. Gonçalves, C.S. Peixoto and L. Dal Bosco et al., 2018. Toxicological assessment of PEGylated single-walled carbon nanotubes in early developing zebrafish. Toxicol. Applied Pharmacol., 347: 54-59.

DOI: 10.1016/j.taap.2018.03.031

Da Rocha, A.M., L.W. Kist, E.A. Almeida, D.G.H. Silva and C.D. Bonan et al., 2018. Neurotoxicity in zebrafish exposed to carbon nanotubes: Effects on neurotransmitters levels and antioxidant system. Comparative Biochem. Physiol., 218: 30-35.

DOI: $10.1016 /$ j.cbpc.2018.12.008

Da Silva, G.H., Z. Clemente, L.U. Khan, F. Coa and L.L.R. Neto et al., 2018. Toxicity assessment of $\mathrm{TiO}_{2}$-MWCNT nanohybrid material with enhanced photocatalytic activity on Danio rerio (Zebrafish) embryos. Ecotoxicol. Environ. Safety, 165: 136-143. DOI: 10.1016/j.ecoenv.2018.08.093

Drobne, D., 2018. Spotlighting CLH report for $\mathrm{TiO}_{2}$ : Nano-safety perspective. Chem. Eng. J., 340: 192-195. DOI: 10.1016/j.cej.2018.01.007

Du, J., J. Cai, S. Wang and H. You, 2017. Oxidative stress and apotosis to zebrafish (Danio rerio) embryos exposed to Perfluorooctane Sulfonate (PFOS) and $\mathrm{ZnO}$ nanoparticles. Int. J. Occupat. Med. Environ. Health, 30: 213-229.

DOI: $10.13075 /$ ijomeh.1896.00669

Du, J., J. Tang, S. Xu, J. Ge and Y. Dong et al., 2018. Parental transfer of perfluorooctane sulfonate and $\mathrm{ZnO}$ nanoparticles chronic co-exposure and inhibition of growth in F1 offspring. Regulatory Toxicol. Pharmacol., 98: 41-49. DOI: 10.1016/j.yrtph.2018.07.005

FDA, 2013. Zebrafish make a splash in FDA research. FDA Consumer Health Information.

Gao, M., M. Lv, Y. Liu and Z. Song, 2018a. Transcriptome analysis of the effects of $\mathrm{Cd}$ and nanomaterial-loaded $\mathrm{Cd}$ on the liver in zebrafish. Ecotoxicol. Environ. Safety, 164: 530-539. DOI: 10.1016/j.ecoenv.2018.08.068

Gao, M., Y. Yang, M. Lv, W. Song and Z. Song, $2018 \mathrm{~b}$. Oxidative stress and DNA damage in zebrafish liver due to hydroxyapatite nanoparticles-loaded cadmium. Chemosphere, 202: 498-505.

DOI: 10.1016/j.chemosphere.2018.03.146

García-Cambero, J.P., M. Núñez-García, G.D. López, A.L. Herranz and L. Cuevas et al., 2013. Converging hazard assessment of gold nanoparticles to aquatic organisms. Chemosphere, 93: 1194-1200. DOI: 10.1016/j.chemosphere.2013.06.074

Gautam, P.K., A. Singh, K. Misra, A.K. Sahoo and S.K Samanta, 2019. Synthesis and applications of biogenic nanomaterials in drinking and wastewater treatment. J. Environ. Manage., 231: 734-748. DOI: $10.1016 /$ j.jenvman.2018.10.104 
González, E.A., D.R. Carty, F.D. Tran, A.M. Cole and P.J. Lein, 2018. Developmental exposure to silver nanoparticles at environmentally relevant concentrations alters swimming behavior in zebrafish (Danio rerio). Environ. Toxicol. Chem., 37: 3018-3024. DOI: 10.1002/etc.4275

Gupta, G.S., A. Dhawan and R. Shanker, 2016. Montmorillonite clay alters toxicity of silver nanoparticles in zebrafish (Danio rerio) eleutheroembryo. Chemosphere, 163: 242-251. DIO: 10.1016/j.chemosphere.2016.08.032

$\mathrm{Hu}$, Q., F. Guo, F. Zhao and Z. Fu, 2017. Effects of titanium dioxide nanoparticles exposure on Parkinsonism in zebrafish larvae and PC12. Chemosphere, 173: 373-379.

DOI: 10.1016/j.chemosphere.2017.01.063

Hou, J., L. Wang, C. Wang, S. Zhang and H. Liu et al., 2018. Toxicity and mechanisms of action of titanium dioxide nanoparticles in living organisms. J. Environ. Sci., 75: 40-53. DOI: $10.1016 /$ j.jes.2018.06.010

Kabir, E., V. Kumar, K.H. Kim, A.C.K. Yip and J.R. Sohn, 2018. Environmental impacts of nanomaterials. J. Environ. Manage., 225: 261-271. DOI: $10.1016 /$ j.jenvman.2018.07.087

Kalueff, A.V., A.M. Stewart and R. Gerlai, 2014. Zebrafish as an emerging model for studying complex brain disorders. Trends Pharmacol. Sci., 35: 63-75. DOI: 10.1016/j.tips.2013.12.002

Keller, A.A., A.S. Adeleye, J.R. Conway, K.L. Garner and L. Zhao et al., 2017. Comparative environmental fate and toxicity of copper nanomaterials. NanoImpact, 7: 28-40.

DOI: 10.1016/j.impact.2017.05.003

Kim, K.T., T. Zaikova, J.E. Hutchison and R.L. Tanguay, 2013. Gold nanoparticles disrupt zebrafish eye development and pigmentation. Toxicol. Sci., 133: 275-288. DOI: 10.1093/toxsci/kft081

Kim, R.O., J.S. Choi, B.C. Kim and W.K. Kim, 2016. Comparative analysis of transcriptional profile changes in larval zebrafish exposed to zinc oxide nanoparticles and zinc sulfate. Bull. Environ. Contaminat. Toxicol., 98: 183-189.

DOI: $10.1007 / \mathrm{s} 00128-016-1995-0$

Kotil, T., C. Akbulut and N.D. Yön, 2017. The effects of titanium dioxide nanoparticles on ultrastructure of zebrafish testis (Danio rerio). Micron, 100: 38-44. DOI: 10.1016/j.micron.2017.04.006

Kteeba, S.M., H.I. El-Adawi, O.A. El-Rayis, A.E. ElGhobashy and J.L. Schuld et al., 2017. Zinc oxide nanoparticle toxicity in embryonic zebrafish: Mitigation with different natural organic matter. Environ. Pollut., 230: 1125-1140.

DOI: $10.1016 /$ j.envpol.2017.07.042
Kteeba, S.M., A.E. El-Ghobashy, H.I. El-Adawi, O.A. El-Rayis and V.S. Sreevidya et al., 2018. Exposure to $\mathrm{ZnO}$ nanoparticles alters neuronal and vascular development in zebrafish: Acute and transgenerational effects mitigated with dissolved organic matter. Environ. Pollut., 242: 433-448.

DOI: $10.1016 /$ j.envpol.2018.06.030

Kumar, P.S.S.R. and S.J. Alexis, 2019. Synthesized Carbon Nanotubes and Their Applications. In: Carbon-Based Nanofillers and Their Rubber Nanocomposites, Yaragalla, S., R.K. Mishra, S. Thomas, N. Kalarikkal and H.J. Maria (Eds.), Elsevier, Amsterdan, pp: 109-122.

Lacave, J.M., Á. Fanjul, E. Bilbao, N. Gutierrez and I. Barrio et al., 2017. Acute toxicity, bioaccumulation and effects of dietary transfer of silver from brine shrimp exposed to PVP/PEI-coated silver nanoparticles to zebrafish. Comparative Biochem. Physiol. C, 199: 69-80.

DOI: $10.1016 /$ j.cbpc.2017.03.008

Lakshmi, K., K. Kadirvelu and P. Mohan, 2019. Reclaimable La: $\mathrm{ZnO} / \mathrm{PAN}$ nanofiber catalyst for photodegradation of methyl paraoxon and its toxicological evaluation utilizing early life stages of zebra fish (Danio rerio). Chem. Eng. J., 357: 724-736. DOI: 10.1016/j.cej.2018.09.201

Lee, W.S., H.J. Cho, E. Kim, Y.H. Huh and H.J. Kim et al., 2019. Bioaccumulation of polystyrene nanoplastics and their effect on the toxicity of $\mathrm{Au}$ ions in zebrafish embryos. Nanoscale, 11: 3173-3185. DOI: $10.1039 / \mathrm{c} 8 \mathrm{nr} 09321 \mathrm{k}$

LeMoine, C.M.R., B.M. Kelleher, R. Lagarde, C. Northam and O.O. Elebute et al., 2018. Transcriptional effects of polyethylene microplastics ingestion in developing zebrafish (Danio rerio). Environ. Pollut., 243: 591-600. DOI: $10.1016 /$ j.envpol.2018.08.084

Li, Y., B. Men, Y. He, H. Xu and M. Liu et al., 2017. Effect of single-wall carbon nanotubes on bioconcentration and toxicity of perfluorooctane sulfonate in zebrafish (Danio rerio). Sci. Total Environ., 607-608: 509-518.

DOI: $10.1016 /$ j.scitotenv.2017.06.140

Liu, H., X. Wang, Y. Wu, J. Hou and S. Zhang et al., 2019. Toxicity responses of different organs of zebrafish (Danio rerio) to silver nanoparticles with different particle sizes and surface coatings. Environ. Pollut., 246: 414-422. DOI: $10.1016 /$ j.envpol.2018.12.034

Liu, Y., Y. Nie, J. Wang, J. Wang and X. Wang et al., 2018. Mechanisms involved in the impact of engineered nanomaterials on the joint toxicity with environmental pollutants. Ecotoxicol. Environ. Safety, 162: 92-102.

DOI: 10.1016/j.ecoenv.2018.06.079 
Ma, Y.B., C.J. Lu, M. Junaid, P.P. Jia and L. Yang et al., 2018. Potential Adverse Outcome Pathway (AOP) of silver nanoparticles mediated reproductive toxicity in zebrafish. Chemosphere, 207: 320-328. DOI: 10.1016/j.chemosphere.2018.05.019

Merrifield, D.L., B.J. Shaw, G.M. Harper, I.P. Saoud and S.J. Davies et al., 2013. Ingestion of metalnanoparticle contaminated food disrupts endogenous microbiota in zebrafish (Danio rerio). Environ. Pollut., 174: 157-163.

DOI: 10.1016/j.envpol.2012.11.017

Misra, A.J., S. Das, A.P. Habeeb-Rahman, B. Das and R. Jayabalan et al., 2018. Doped $\mathrm{ZnO}$ nanoparticles impregnated on Kaolinite (Clay): A reusable nanocomposite for photocatalytic disinfection of multidrug resistant Enterobacter sp. under visible light. J. Colloid Interface Sci., 530: 610-623.

DOI: $10.1016 /$ j.jcis.2018.07.020

Morozesk, M., L.S. Franqui, A.S. Mansano, D.S.T. Martinez and M.N. Fernandes, 2018. Interactions of oxidized multiwalled carbon nanotube with cadmium on zebrafish cell line: The influence of two co-exposure protocols on in vitro toxicity tests. Aquatic Toxicol., 200: 136-147.

DOI: $10.1016 /$ j.aquatox.2018.05.002

Naasz, S., R. Altenburger and D. Kühenl, 2018. Environmental mixtures of nanomaterials and chemicals: The Trojan-horse phenomenon and its relevance for ecotoxicity. Sci. Total Environ., 635: 1170-1181. DOI: 10.1016/j.scitotenv.2018.04.180

Oksel, C., C.Y. Ma and X.Z. Wang, 2015. Structureactivity relationship models for hazard assessment and risk management of engineered nanomaterials. Proc. Eng., 102: 1500-1510.

DOI: $10.1016 /$ j.proeng.2015.01.284

Orbea, A., N. González-Soto, J.M. Lacave, I. Barrio and M.P. Cajaraville, 2017. Developmental and reproductive toxicity of PVP/PEI-coated silver nanoparticles to zebrafish. Comparative Biochem. Physiol., 199: 59-68. DOI: 10.1016/j.cbpc.2017.03.004

Pitt, J.A., J.S. Kozal, N. Jayasundara, A. Massarsky and R.O. Trevisan et al., 2018a. Uptake, tissue distribution and toxicity of polystyrene nanoparticles in developing zebrafish (Danio rerio). Aquatic Toxicol., 194: 185-194. DOI: 10.1016/j.aquatox.2017.11.017

Pitt, J.A., R.O. Trevisan, A. Massarsky, J.S. Kozal and E.D. Levin et al., 2018b. Maternal transfer of nanoplastics to offspring in zebrafish (Danio rerio): A case study with nanopolystyrene. Sci. Total Environ., 643: 324-334.

DOI: $10.1016 /$ j.scitotenv.2018.06.186

Ponnamma, D., J.J. Cabibihan, M. Rajan, S.S. Pethaiah and K. Deshmukh et al., 2019. Synthesis, optimization and applications of $\mathrm{ZnO} /$ polymer nanocomposites. Mater. Sci. Eng.

DOI: $10.1016 /$ j.msec.2019.01.081
Ramachandran, R., C. Krishnaraj, V.K.A. Kumar, S.L. Harper and T.P. Kalaichelvan et al., 2018. In vivo toxicity evaluation of biologically synthesized silver nanoparticles and gold nanoparticles on adult zebrafish: A comparative study. 3 Biotech, 8: 441-441. DOI: $10.1007 / \mathrm{s} 13205-018-1457-y$

Ramachandran, R., C. Krishnaraj, A.S. Sivakumar, P. Prasannakumar and V.K. Abhay-Kumar et al., 2017. Anticancer activity of biologically synthesized silver and gold nanoparticles on mouse myoblast cancer cells and their toxicity against embryonic zebrafish. Mater. Sci. Eng., 73: 674-683.

DOI: $10.1016 /$ j.msec.2016.12.110

Ren, X., X. Zhao, X. Duan and Z. Fang, 2018. Enhanced bio-concentration of tris(1,3-dichloro-2-propyl) phosphate in the presence of nano- $\mathrm{TiO}_{2}$ can lead to adverse reproductive outcomes in zebrafish. Environ. Pollut., 233: 612-622. DOI: $10.1016 /$ j.envpol.2017.10.101

Rotomskis, R., Ž. Jurgelènè, M. Stankevičius, M. Stankevičiūtè and N. Kazlauskienè et al., 2018. Interaction of carboxylated $\mathrm{CdSe} / \mathrm{ZnS}$ quantum dots with fish embryos: Towards understanding of nanoparticles toxicity. Sci. Total Environ., 635: 1280-1291. DOI: 10.1016/j.scitotenv.2018.04.206

Sarkar, B., S.K. Verma, J. Akhtar, S.P. Netam and S.K. Gupta et al., 2018. Molecular aspect of Silver nanoparticles regulated embryonic development in Zebrafish (Danio rerio) by Oct-4 expression. Chemosphere, 206: 560-567.

DOI: 10.1016/j.chemosphere.2018.05.018

Sleight, V.A., A. Bakir, R.C. Thompson and T.B. Henry, 2017. Assessment of microplastic-sorbed contaminant bioavailability through analysis of biomarker gene expression in larval zebrafish. Marine Pollut. Bull., 116: 291-297.

DOI: 10.1016/j.marpolbul.2016.12.055

Strungaru, S.A., G. Plavan, A. Ciobica, M. Nicoara and M.A. Robea et al., 2018. Acute exposure to gold induces fast changes in social behavior and oxidative stress of zebrafish (Danio rerio). J. Trace Elements Med. Biol., 50: 249-256.

DOI: $10.1016 /$ j.jtemb.2018.07.013

Sung, H.K., E. Jo, E. Kim, S. Yoo and J. Lee et al., 2018. Analysis of gold and silver nanoparticles internalized by zebrafish (Danio rerio) using single particle-inductively coupled plasma-mass Spectrometry. Chemosphere, 209: 815-822. DOI: $10.1016 /$ j.chemosphere.2018.06.149

Thit, A., L.M. Skjolding, H. Selck and J. Sturve, 2017. Effects of copper oxide nanoparticles and copper ions to zebrafish (Danio rerio) cells, embryos and fry. Toxicol. Vitro, 45: 89-100. DOI: $10.1016 /$ j.tiv.2017.08.010 
Tian, J., J. Hu, G. Liu, H. Yin and M. Chen et al., 2019. Altered gene expression of $\mathrm{ABC}$ transporters, nuclear receptors and oxidative stress signaling in zebrafish embryos exposed to CdTe quantum dots. Environ. Pollut., 244: 588-599. DOI: 10.1016/j.envpol.2018.10.092

Vale, G., K. Mehennaoui, S. Cambier, G. Libralato and S. Jomini et al., 2016. Manufactured nanoparticles in the aquatic environment-biochemical responses on freshwater organisms: A critical overview. Aquatic Toxicol., 170: 162-174.

DOI: 10.1016/j.aquatox.2015.11.019

Van Pomeren, M., N.R. Brun, W.J.G.M. Peijnenburg and M.G. Vijver, 2017. Exploring uptake and biodistribution of polystyrene (nano)particles in zebrafish embryos at different developmental stages. Aquatic Toxicol., 190: 40-45. DOI: $10.1016 /$ j.aquatox.2017.06.017

Verma, S.K., E. Jha, P.K. Panda, M. Mukherjee and A. Thirumurugan et al., 2018. Mechanistic insight to ROS and neutral lipid alteration induced toxicity in human model with fins (Danio rerio) by industrially synthesized Titanium dioxide nanoparticles. Toxicol. Res., 7: 244-257. DOI: 10.1039/C7TX00300E

Wang, S., C. Zhuang, J. Du, C. Wu and H. You, 2017. The presence of MWCNTs reduces developmental toxicity of PFOS in early life stage of zebrafish. Environ. Pollut., 222: 201-209. DOI: 10.1016/j.envpol.2016.12.055

Williams, R.J., S. Harrison, V. Keller, J. Kuenen and S. Lofts et al., 2019. Models for assessing engineered nanomaterial fate and behaviour in the aquatic environment. Curr. Opin. Environ. Sustainability, 36: 105-115. DOI: 10.1016/j.cosust.2018.11.002

$\mathrm{Xu}$, J., R. Zhang, T. Zhang, G. Zhao and Y. Huang et al., 2017. Copper impairs zebrafish swimbladder development by down-regulating Wnt signaling. Aquatic Toxicol., 192: 155-164.

DOI: $10.1016 /$ j.aquatox.2017.09.018
Yan, Z., H. Sun, R. Jiang, H. Dong and H. Yang et al., 2018a. Accumulation, metabolite and active defence system responses of fluoxetine in zebrafish embryos: Influence of multiwalled carbon nanotubes with different functional groups. Aquatic Toxicol., 205: 204-212. DOI: 10.1016/j.aquatox.2018.10.018

Yan, Z., Y. Liu, H. Sun and G. Lu, 2018b. Influence of multiwall carbon nanotubes on the toxicity of $17 \beta$ estradiol in the early life stages of zebrafish. Environ. Sci. Pollut. Res., 25: 7566-7574. DOI: $10.1007 / \mathrm{s} 11356-017-1063-2$

Ye, N., Z. Wang, S. Wang and W.J.G.M. Peijnenburg, 2018. Toxicity of mixtures of zinc oxide and graphene oxide nanoparticles to aquatic organisms of different trophic level: Particles outperform dissolved ions. Nanotoxicology, 12: 423-438. DOI: $10.1080 / 17435390.2018 .1458342$

Zarco-Fernández, S., A.M. Coto-García, R. MuñozOlivas, J. Sanz-Landaluze and S. Rainieri et al., 2016. Bioconcentration of ionic cadmium and cadmium selenide quantum dots in zebrafish larvae. Chemosphere, 148: 328-335.

DOI: 10.1016/j.chemosphere.2015.12.077

Zhang, Y., Z. Ding, G. Zhao, T. Zhang and Q. Xu et al., 2018. Transcriptional responses and mechanisms of copper nanoparticle toxicology on zebrafish embryos. J. Hazardous Mater., 344: 1057-1068. DOI: $10.1016 /$ j.jhazmat.2017.11.039

Zhao, X., X. Ren, R. Zhu, Z. Luo and B. Ren, 2016. Zinc oxide nanoparticles induce oxidative DNA damage and ROS-triggered mitochondria-mediated apoptosis in zebrafish embryos. Aquatic Toxicol., 180: 56-70. DOI: 10.1016/j.aquatox.2016.09.013 